evolutionary explanation of diversity, are invariably interpreted using methods which presuppose, but do not demonstrate, evolutionary relationships, and which use criteria that are essentially functional and teleological. Finally, there is a collection of isolated fragmentary pieces of evidence which are usually dismissed as anecdotal because they are irreconcilable with the evolutionary model. From the above and other considerations it is possible to argue a strong case for special creation.

If, however, we are to be banished to the lunatic fringe, we find ourselves in the company of men who are regarded as the founders of modern science - Boyle, Newton et al. These men formulated the laws of physics and chemistry, not by invoking the supernatural but by assuming it, in the conviction that all things were made by an intelligent and all-powerful Creator who had imposed order and meaning on his creation. So it seemed to them, and is it not reasonable to ask whether they would have begun to ask the right questions if it had seemed otherwise? It has, for example, been suggested by

Needham that the reason for the failure of the Chinese to develop the scientific method of the West was that they did not see order in nature as ordained by a personal, rational being.

Science can be conducted only within a context or "world view" which inescapably determines its aims and directions. We, as scientists, must be aware that as long as our society looks to the great god of science for direction and meaning, our own world view will be shaped by our own science. If we assert untruth, half-truth or hypothesis as fact, society and we ourselves will be misled and we will be responsible for the cultural, political and ethical consequences. Truth was never determined by majority opinion.

We are dismayed by the methods and attitudes of American Creationism. The legislature cannot be used to establish truth, either scientific or spiritual. Furthermore we believe that the Bible, though accurate, was not written as a scientific textbook but written primarily to lead men to a personal knowledge of God through His Son. In that knowledge we are able to say: "By faith we understand that the world was created by the word of God"' (Hebrews 11.3).

Chris Darnbrough JOHN GODDARD University of Glasgow, UK William S. Stevely

\section{Room for faith}

SIR - Contrary to what your editorial on creationism and evolution might suggest (Nature 28 May, p.271), few "card-carrying believers" within the scientific community would hold to the "God of gaps" theory you describe. Few see any major conflict between science and religion. Most see them as providing different languages to describe the same phenomenon - the view that God created the world and that evolution was the mechanism by which he did it is not contradictory. "God created the world" cannot be held up as a scientific theory. It is scientifically and theologically unprovable. It has to remain a matter of faith. However, some of the corollaries of creationism: the insufficiency of mutation and natural selection to account for intra-specific diversity, catastrophic geology, a shrinking rather than an expanding active gene pool; are worthy of some consideration within a scientific context. Indeed, it is surprising how rapidly some tentative theories in support of evolution have become scientific facts. The absurdity of recapitulation and the half-truth of horse evolution are still incorporated into school textbooks as irrefutable fact. Evolutionists often present their "beliefs" as dogmatic "fact" in a similar vein to creationists.

What is required is not that creation should gain equal time within the school curriculum of Arkansas or the exhibitions of the British Museum (Natural History); but that school pupils and general public alike are presented with the solid facts as far as they are known with both their limitations and their certainties (but not with conjecture or extrapolation). And that they be encouraged to draw their own conclusions; to observe the data, to construct their hypothesis and to test it against the new data as they emerge. In other words, they should be encouraged to learn and apply the scientific method. This should be the basis of all biology courses and museum exhibitions.

However, the controversy will not be stayed by the application of scientific method; because what one believes about the origin of life and of man has a grave effect on how one treats fellow man and how society functions.

Microbiology Department,

NeIL K. MCBride

Queen Elizabeth College, London W8, UK

\section{Missing links}

$\mathrm{SIR}$ - In your leading article "How true is the theory of evolution?" (Nature 12 March, p.75), you discussed the apparent inability of the Darwinian theory of evolution controlled by natural selection to explain the episodic nature of the fossil record.

It must be accepted that the fossil record of most evolutionary lines does indeed show changes taking place in discontinuous jumps rather than in a continuous manner. It is important to realize that even a continuous development resulting from the accumulation of many small changes may only rarely show itself as this in the fossils unearthed.

We are unlikely to find the fossil remains of more than one in a hundred million or so individuals. Most of the fossils which we see, therefore, are necessarily those of organisms which were common over large areas and which remained common and unchanged for long periods. For this to be possible the species concerned must have been well adapted to a particular niche in a stable environment. If the environment changes the niche must change, and may then be invaded successfully by another species, or even vanish altogether.

Once a species has filled a stable niche it can rarely gain by changing in anything but minor ways. In an environment which has been stable for a long time, all major neighbouring niches are likely to be filled by other successful organisms, so that individuals that differ from the species optimum are likely to be less successful than the norm. Natural selection will then act to slow down significant evolutionary change.

Rapid evolution can occur only where there is extremely intense intraspecific competition, as in the case of our own ancestors, or where a species is not well adapted to its habitat - for example in regions colder or wetter than the stable environment to which it was adapted, and/or with different fauna or flora.

Population pressure in a successful species will constantly be supplying a trickle of individuals to such non-optimal peripheral regions around the main habitat. In such a region a small mutation, useless or even mildly harmful, could be advantageous to survival outside the normal range. Again, this would be unlikely to help if the new area itself had a stable climax ecology where all important niches were already filled. It is in a small area with many empty niches, and perhaps cut off by some natural barrier introduced by climatic, volcanic or other phenomena, that a now nonoptimally adapted species may survive and find a whole series of minor mutations advantageous, eventually leading to major changes in structure and behaviour. During the whole of this development numbers will be limited, allowing a significant degree of genetic drift, and the intermediate forms may not persist for long, so that the chances of our finding fossils of such intermediates will be slight.

Not until the species has changed so much that it can successfully invade a new stable area when the barriers break down, as the rabbit did in Australia, can it become common and persistent over an extended period - and give us a chance to find its remains.

I am not trying to disprove the occurrence at some times in the past of large discontinuous jumps in evolution, or to prove that all evolution has occurred as a result of natural selection acting on a large number of random mutations, mostly small. All I am saying is that whichever of these is true the fossil record must be expected to be discontinuous.

"Missing links" may be truly missing, or simply so small in number and period of existence that they have not been found.

J.H. FremLiN

Department of Physics, University of Birmingham, UK

\section{Scientists for sale?}

SIR - Increasingly, the members of the biomedical research community are being forced to give their activities a greater immediacy of application. The commercial pattern into which the work on genetic engineering and monoclonal antibodies is falling reflects this pressure. There could arise conflicts in the academic mind some of which merit public discussion.

In the past there have been no constraints, other than moral, on the free movement of individuals between research groups.

However, now, many of the commercially useful activities, whether in industrial or nonindustrial laboratories, involve team work. These groups, like football teams, require continuity for success and their members, it can be argued, should be subject to the same restraints on movement from laboratory to laboratory as are footballers between teams. Specifically, contracts of employment should be considered as not only binding on the employer but also on the employee. It could even be maintained that changes within the period of a contract could be arranged by mutual agreement but only on payment of an appropriate transfer fee. Breaking of the contract by either employer or employee should be subject to the process of the law in the usual way.

As things stand at the moment, poaching of staff and of ideas on which considerable development money has been spent can occur without let or hindrance.

A.J.S. DAvies

Institute of Cancer Research,

Royal Cancer Hospital, London, UK 